
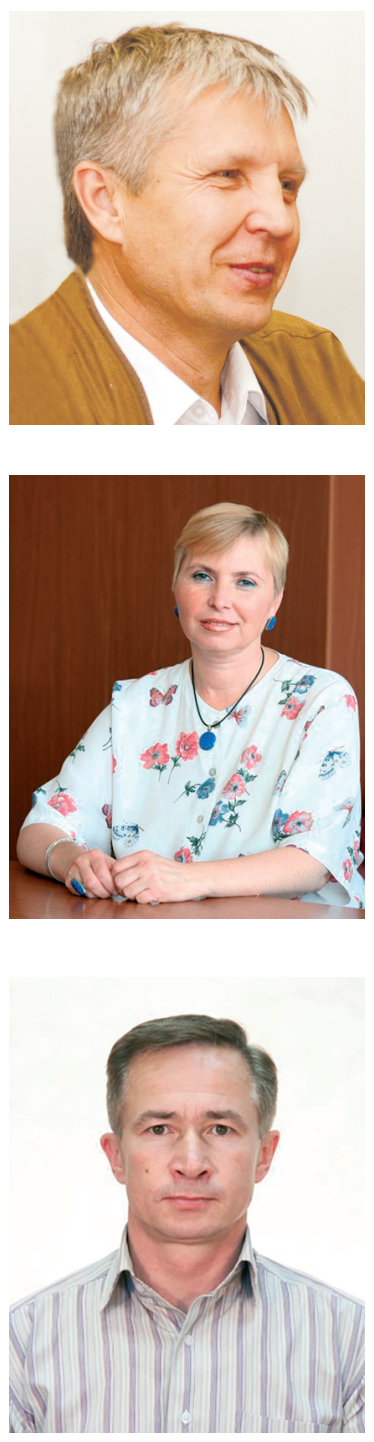

\section{Суходолов Александр Петрович}

доктор экономических наук, профессор, ректор

Байкальский государственный университет,

664003, Российская Федерация, г. Иркутск, ул. Ленина, 11, e-mail: rector@bgu.ru

\section{Alexander P. Sukhodolov \\ DPhil. in Economics, Professor, Rector \\ Baikal State University, \\ 11 Lenin St., 664003, Irkutsk, Russian Federation, \\ e-mail: rector@bgu.ru}

\section{Кузнецова Ирина Альфредовна \\ Кандидат технических наук, доцент}

кафедра информатики и кибернетики

Байкальский государственный университет,

664003, Российская Федерация, г. Иркутск, ул. Ленина, 11, e-mail: iak_irk@bk.ru

\section{Irina A. Kuznetsova}

$\mathrm{PhD}$ in Engineering, Associate Professor

Computer Science \& Cybernetics Department, Baikal State University, 11 Lenin St., Irkutsk, 664003, Russian Federation, e-mail: iak_irk@bk.ru

\section{Тимофеев Сергей Викторович}

Кандидат физико-математических наук, доцент

кафедра математики и эконометрики,

Байкальский государственный университет, Российская Федерация, 664003, г. Иркутск, ул. Ленина, 11, e-mail: timofeevsv12@gmail.com

\section{Sergey V. Timofeev}

Phd in Physics and Mathematics, Associate Professor Mathematics \& Econometrics Department, Baikal State University, 11 Lenin St., 664003, Irkutsk, Russian Federation, e-mail: timofeevsv12@gmail.com

\title{
АНАЛИЗ ПОДХОДОВ В МОДЕЛИРОВАНИИ СРЕДСТВ МАССОВОЙ ИНФОРМАЦИИ
}

Аннотация. Под СМИ в рамках данного исследования понимаются социальные институты, в фрункции которых входят сбор, обработка, анализ и распространение информации в массы с целью определенного воздействия на сознание людей.

В настоящее время развитие системных исследований в области коммуникационных процессов дает возможность, с одной стороны, те- 
оретически обосновать новые подходы и методы изучения этих процессов, с другой, определить пути повышения эффективности использования полученных результатов при решении актуальных практических задач.

В статье особое внимание уделено роли имитационного моделирования для описания изучаемой системы-оригинала системой-заменителем с целью изучения первой в конкретных аспектах.

Представлен обзор концептуальных моделей средств массовой информации (коммуникации) с проведением сравнительного анализа их теоретической и практической значимости в процессах управления, достоинств и недостатков.

Также дан обзор и анализ появившихся в последнее время в научных журналах математических моделей, при помощи которых сделана попытка описания закономерностей функционирования и развития СМИ как динамической системы. Предлагаемые подходы описаны с привлечением аппарата обыкновенных дифференциальных уравнений. Несмотря на некоторое различие в фрормах представления СМИ, во всех рассмотренных моделях отражены инвариантно-структурные отношения, обусловливающие целостность системы.

Авторами указано на необходимость дальнейших предметных исследований для раскрытия других важных сторон реализации коммуникативных процессов, расширяющих представление о гомеостазе - достижении цели функционирования и сохранении целостности.

Статья продолжает тему, поднятую в научном докладе А. П. Суходолова и М. П. Рачкова «К созданию теории средств массовой информации: поставка задачи», сделанном на совместном заседании кафедр журналистики и экономики СМИ, информатики и кибернетики, математики и эконометрики Байкальского государственного университета 26 ноября 2015 г. (см: Вопросы теории и практики журналистики. - 2016. № 1. - C. 6-13).

Ключевые слова. Система, средства массовой информации, анализ, информация, коммуникация, реципиент, модель, имитация, гомеостаз.

Информация о статье. Дата поступления 05 мая 2017 г.; дата принятия к печати 29 мая 2017 г.; дата онлайн-размещения 08 июня 2017 г.

\section{THE ANALYSIS OF APPROACHES IN MODELLING OF MASS MEDIA}

Abstract. In the framework of this research mass media are viewed as social institutions whose functions include collecting, processing, analyzing and disseminating of information to audiences in order to influence their consciousness in a particular way.

Nowadays, developing systemic research in mass communication allows, on the one hand, to theorize new approaches to and methods of studying mass communication processes, and, on the other hand, to specify ways of increasing effectiveness of using research findings while doing essential practical tasks.

The article thoroughly considers the role of simulation modeling for describing an original system under study via a substitute system in order to study the original one from certain angles. 
Conceptual models of mass media are considered, including comparative analysis of their theoretical and practical value in management processes, as well as these models' advantages and drawbacks.

Also, the article considers mathematical models taken from recent journals' publications which are used to describe patterns of functioning and developing of mass media as a dynamic system. The approaches proposed are described with the help of simple differential equations. Despite some differences related to forms of representing mass media, all the models under consideration show invariant-structural relations ensuring the system's integrity.

The authors state the necessity of further research into this subject in order to develop other significant aspects of realization of communicative processes, broadening one's knowledge of homeostasis - achieving the goal of functioning and ensuring integrity.

The article develops the issue raised in Alexander P. Sukhodolov and Mikhail P. Rachkov's speech at a collaborative meeting of Department of Journalism and Economics of Mass Media, Department of Computer Science and Cybernetics, Department of Mathematics and Econometrics of Baikal State University on 26 November 2015. (See Theoretical and Practical Issues of Journalism. - 2016. - № 1. P. 6-13).

Keywords. System, mass media, analysis, information, communication, recipient, model, simulation, homeostasis.

Article info. Received May 05, 2017; accepted May 29, 2017; available online June 08, 2017.

Введение. Средства массовой информации (СМИ) - сложная динамическая система, формирующая целенаправленный объективно-субъективный фрактор для функционирования и развития социальной сферы. Субстратом СМИ является информация, которая определяет состояние общества в рассматриваемый временной период в экономическом, политическом, культурном и прочих аспектах.

Системный подход и системное мышление как универсальный метод познания дает инструмент исследования сложных объектов, в том числе социальных, способствует анализу информационных процессов и совершенствованию технологий использования информации в решении задач в области коммуникаций. Системное представление СМИ обусловливает применение спосо- бов и средств системного подхода, позволяющего раскрыть суть коммуникации, информационного воздействия на людей, формирующего и преобразующего их менталитет.

\section{Роль имитационного модели- рования в изучении систем}

Системное исследование, по мнению многих ученых, например, А. А. Богданова [1], Л. фон Берталанфри [2] и др., играет основополагающую роль для комплексного описания сложных общественных процессов. С одной стороны, формируются общетеоретические знания, необходимые для создания познавательного базиса, с другой, специально-научные и практические - для объяснения явлений системности в обществе с различных позиций. Последние тесно связаны с научно-технической революцией, математизацией, 
инженеризацией, кибернетизацией науки и практической деятельности.

Основоположник тектологии (тектология с греч. - учение о строительстве) Богданов утверждал: организации систем разных уровней тождественны - от микромира до социальных систем. В социальных процессах, по его мнению, «всякая человеческая деятельность объективно является организующей или дезорганизующей, то есть любую человеческую деятельность можно рассматривать как некоторый материал организационного опыта и исследовать с организационной точки зрения» [1].

В рамках общей теории систем Берталанфи считал основными задачами следующие:

- установление общих принципов и законов поведения систем любой природы;

- выявление в биологических и социальных объектах законов, аналогичных законам естествознания;

- синтез современного научного знания на основе выявления изоморфизма законов различных сфер деятельности.

Наряду с такими важными системными принципами, как господство целого над частным, эмерджентность, структурность и ее инвариантность, иерархичность, разнообразие состояний и пр., Берталанфи уделял внимание свойству системы сохранять жизненно важные параметры при изменении условий функционирования для поддержания своего существования, то есть гомеостазу.

Самым важным для социальной системы является эффрективное управление. Высокая степень сложности заключается в двойственной природе человека, у которого личные интересы вступают в противоречие с общественными.

Любое исследование требует специального инструмента, позволяющего испытать теоретические законы на практике, не проводя эксперименты с реальными системами. Зачастую это невозможно, поэтому традиционно применяется имитационное моделирование [3-6].

Под имитацией будем понимать процесс представления изучаемой системы-оригинала системой-заменителем с целью изучения первой. Термин «имитация» является, по сути, синонимичным термину «моделирование» с той лишь разницей, что первый в настоящее время связан с использованием компьютерных технологий.

Преимуществами имитации в сравнении с другими методами исследования отметим следующие:

- имитация позволяет экспериментально исследовать сложные внутренние взаимодействия в рассматриваемой системе;

- имеется возможность вносить организационные и информационные изменения в модель;

- имитация помогает более детально изучить поведение системы и ее элементов с тем, чтобы улучшить качество ее функционирования;

- имитационные эксперименты служат базой для обучения студентов и молодых специалистов;

- имитационные эксперименты могут помочь исследователю разобраться в том, какие из учитываемых фракторов наиболее существенны, а какие нет;

- имитацию применяют для изучения новых ситуаций, которые могут иметь место в будущем, отра- 
ботать стратегию и тактику поведения в новых условиях;

- имитация может использоваться для проверки новых стратегий и правил принятия решений перед использованием их в реальной системе;

- имитация позволяет изучать динамику поведения системы в реальном масштабе времени;

- имитация с успехом может быть использована для предсказания «узких мест» и других трудностей, появляющихся в поведении системы при введении в нее новых элементов [3; 4].

\section{Анализ моделей коммуника- ции}

В теории и практике журналистики давно большое внимание уделяется вопросам моделирования коммуникативных отношений. Существует несколько моделей коммуникаций, вскрывающих суть информационного влияния (пропаганды) на получателя в целях переформатирования структуры сознания, ценностей, мироощущения. Несмотря на разнообразие представлений систем каждая из них подчеркивает наличие единства двух сторон отношений источника и потребителя (реципиента) информации, которое обеспечивается специальной системой управления. Далее рассмо- трим самые известные подходы к моделированию процесса коммуникации.

В рамках данного исследования под системой коммуникации будем понимать систему управления отношениями между источником (производителем информации) и потребителем (приемником информационного сообщения), в которой оказывается активное информационное влияние друг на друга.

Модель Шеннона-Уивера [7]. Модель Шеннона-Уивера (рис. 1) представляет схему коммуникационного процесса, является одноканальной линейной и содержит следующие компоненты:

- источник;

- передатчик;

- несущий сигнал;

- приемник;

- получатель.

Кроме этого учтен внешний искажающий сообщение фактор, называемый «шумом», который, повидимому, может оказывать двоякое влияние на получателя. С одной стороны, технические помехи уменьшают семантическое качество сообщения, с другой, вредная или ложная информация, встроенная в сообщение, вступает в противоречие с передаваемой источником информацией и играет роль перестройки цели воздействия на конечное звено.

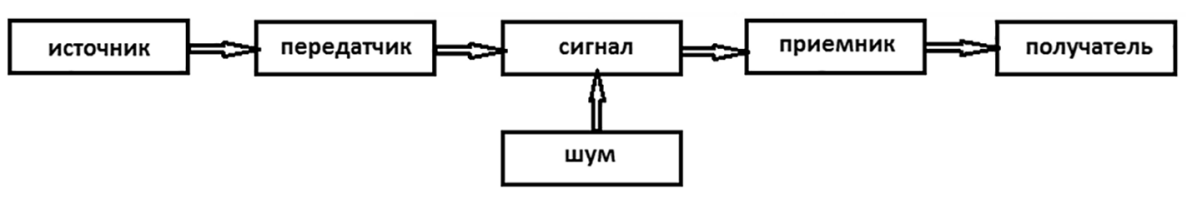

Рис. 1. Универсальная линейная схема процесса коммуникации Шеннона-Уивера 
Достоинством такого подхода является простота представления коммуникационного процесса, так как схема несет образ технического устройства. При этом следует отметить ряд недостатков и возникающих в связи с этим проблем, которые не позволяют использовать модель для раскрытия технологии информационного воздействия на потребителей смысла сообщения.

Проблемы подразделяются на три вида:

- технические, связанные с преодолением технических помех при передаче сигнала - наиболее легкие;

- семантические - из-за различий получателей по социальным группам; психотипическим особенностям воспринимающих субъектов; различий в уровне образования, жизненном опыте и пр.;

- прагматические проблемы, включающие различные виды эмоциональных, эстетических и нравственных реакций, а также пропаганду и связанное с ней манипулирование реципиентом, (наиболее сложные в сфрере социального взаимодействия).

К существенному недостатку схемы следует отнести отсутствие звена, позволяющего регулировать качество процесса передачи сообщения. Это означает отсутствие цепи обратной связи и относит тип коммуникации по такому принципу к категории «информационное агентство».

Модель «Волшебной пули» Лассуэлла. Модель «Волшебной пули» [8; 9] является моделью коммуникативного акта и представлена в 1948 г. представителем Чикагской школы политологов Гаральдом Лассуэлом. По структуре она является линейной, по сути - отражает процесс передачи информационного сообщения от источника реципиенту как летящей пули от стрелка к живой мишени для «волшебной» трансформации идей, чувств, знаний или мотивации получателя. Графически модель воплощается в пятиблочную конструкцию (см. рис. 2). Схема включает следующие компоненты:

- субъект-создатель информационного сообщения, отражающего цель воздействия;

- сообщение;

- канал передачи с кодированием и декодированием;

- субъект-приемник сообщения, который формирует реакцию на информационной воздействие;

- канал обратной связи для сверки цели с результатом информационного воздействия в блоке отправителя.

По сравнению с моделью Шеннона-Уивера данный способ представления процесса коммуникации выделяет фрункцию регулирования за счет замыкания цепи передачи информационного сообщения блоком

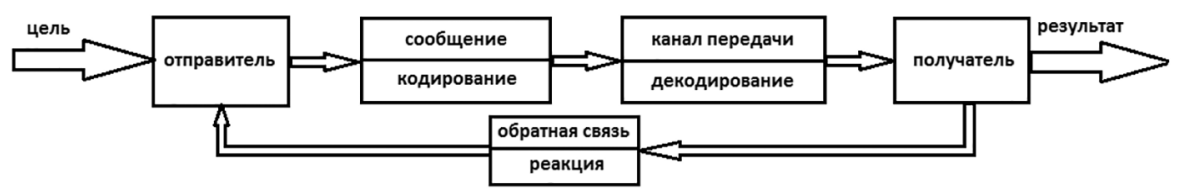

Рис. 2. Линейная модель Лассуэлла 


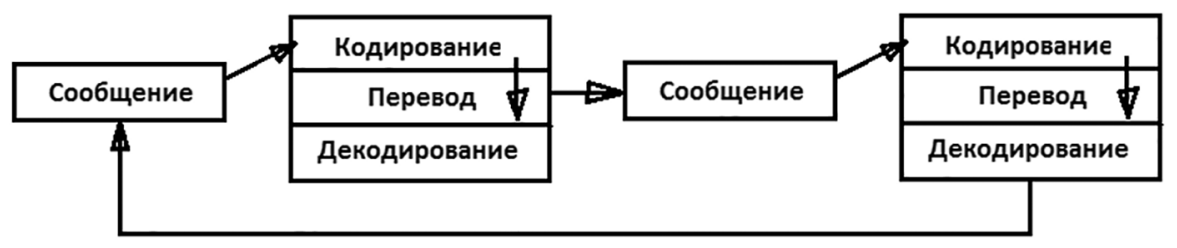

Рис. 3. Модель Шрамма-Осгуда

обратной связи. Роль данного звена заключается в обоснованном регулировании процесса информационного воздействия для исполнения цели информационной системы. В литературных источниках, посвященных теории управления, схема на рис. 2 называется гомеостатом Эшби [10].

Главными достоинствами модели являются универсальность и самоорганизация коммуникационного процесса, следовательно, управляемость согласно поставленной цели. К первому недостатку отнесем «однобокий», безальтернативный характер влияния передаваемого сообщения на получателя. Второй недостаток относится к обратной связи, которая может быть отрицательной, т. е. за счет ее использования система удерживает процесс достижения цели в установленных рамках, а с другой стороны, положительной и приводит получателя к состоянию паники, депрессии и т. п. Требуется включение в схему структурно идентичного информационного «оппонента» - антагониста, который позволит системе коммуникации управлять социальным гомеостазом [11-14].

Циркулярная (циклическая) модель коммуникации Шрамма-Осгуда. Циклическая модель Шрам-
ма-Осгуда [15] на рис. 3 представляет замкнутую последовательность преобразования сообщения. Она демонстрирует реакцию коммуниканта на сообщение в форме обратной связи, формируя диалог, который сводится к консенсусу с удовлетворением целей участников.

Данная модель является замкнутой и нелинейной. Следовательно, нельзя точно установить источник сообщения, который является и получателем. В этом состоит особенность модели и ее недостаток. Кроме того, итерационный обмен информационными сообщениями может быть условно бесконечным, что вносит неопределенность в результате исполненного акта. К преимуществу можно отнести расширение функций: на ряду с наличием обратной связи в системе возможно продемонстрировать диалог участников информационного обмена.

Двухступенчатая модель Сми [15]. Однофразный акт передачи сообщения реципиенту, который представлен в линейных моделях, не является универсальным инструментом, демонстрирующим процесс коммуникации. Это объясняется тем, что информация в форме пере- 
даваемого сообщения не дает одинаковый эффрект воздействия на получателей.

По аналогии с технической системой, для воздействия на управляемый объект необходимо входной сигнал с помощью усилителя преобразовать в другую форму. Так как аудитория имеет разный уровень подготовки к восприятию поступающей информации, необходимо в процессе передачи использовать дополнительное звено - приемник-источник, которое, переводя сообщение в другую форму для аудитории, усиливает эффрект его воздействия. Второе звено рождает новый тип структуры - двухступенчатую. Модели можно расширять, получая многоступенчатые схемы.

Модель двухступенчатого потока предложена Лазарсфельдом и Берельсоном [15]. Преимуществом модели является усиление эфффекта воздействия информации через преобразование сообщения в сообщение, понятное аудитории. Ко второму преимуществу можно отнести упрощение деятельности и повышение результативности массмедиа. В качестве недостатка, на наш взгляд, следует отметить отсут- ствие возможности интерпретировать сообщение с противоположным мнением и доставить результат общественной аудитории. Кроме того, в некоторых случаях сообщения от источника принимаются непосредственно реципиентом, минуя второе звено.

Модель Робинсона [15; 16] расширяет двухступенчатую схему: наряду с активными реципиентами включены пассивные, не воспринимающие сообщение от авторитетов. В данном варианте к недостаткам следует отнести отсутствие методик расчленения реципиентов на активных и пассивных и оценки степени важности участия авторитета в коммуникационном процессе.

Модель Ньюкомбо. Коммуникационная модель Ньюкомбо [17] демонстрирует возникновение коммуникации как нарушение симметрии суждений на некоторую тему $X$ двух $A, B$ и более индивидов (рис. 4). Восстановление симметрии суждений участников происходит за счет обмена информацией. Последняя играет роль, похожую на действие жидкости в сообщающихся сосудах: уравновешивает мнения и сводит к некоторому консенсусу участников обсуждения.

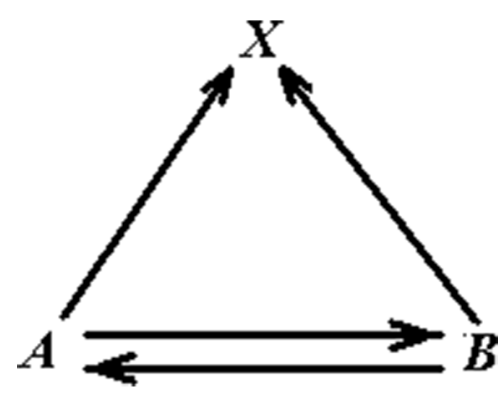

Рис. 4. Модель Ньюкомбо 
Недостатки данного подхода:

- отсутствие правил взаимодействия, ограничивающих дисбаланс мнений;

- игнорирование фактора важности акта обсуждения темы хотя бы одним из участников;

- отсутствие учета компетентности оппонентов.

Для устранения указанных "узких мест» автор включил в модель дополнительные ограничения и условия. Модифицированный вариант может успешно применяться для описания дискуссии единомышленников на политические темы.

Модель Вестли и Маклина [16; 17]. Она является фрункциональной моделью СМИ, расширяет процесс коммуникации, описываемый моделью Ньюкомбо, совмещая межличностную, межгрупповую
К недостатку относится ограниченный выбор доступных объектов внешней среды. Это вносит ограничения в способности воспринимать и обрабатывать информацию.

Модель Малецке [18]. Немецкий ученый Герберт Малецке, опираясь на модель Лассуэлла, рассматривает вместо пяти четыре главных компоненты массовой коммуникации (см. рис. 5):

— коммуникатор К;

- сообщение C;

- СМИ;

- реципиент P.

Автор не ставит во главу угла лассуэлловский «эффрект», не отклоняет задачу его детального анализа. Он считает, что воздействие (складывание эффректа) происходит у того, кто воспринимает

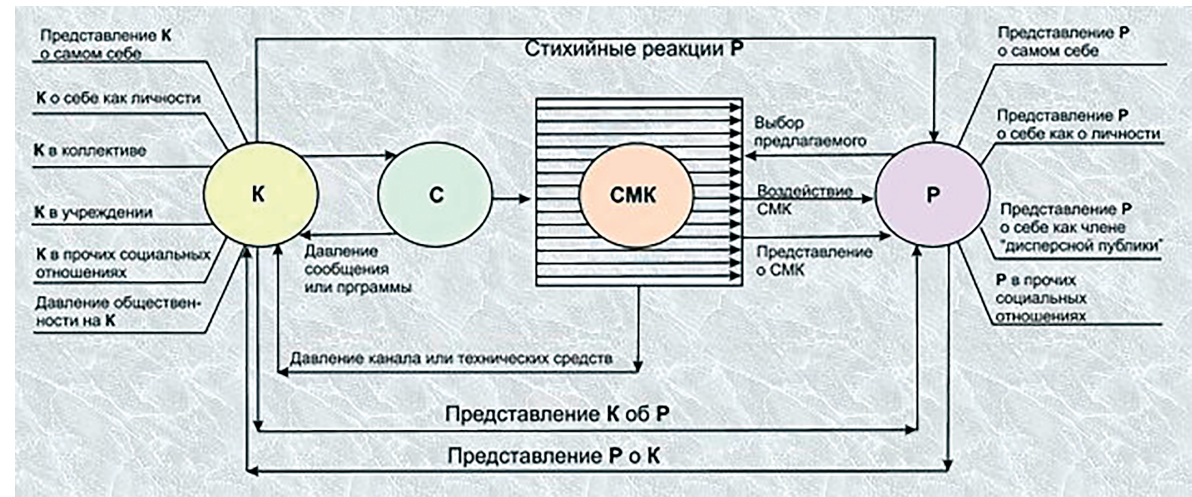

Рис. 5. Модель Малецки:

К - коммуникатор; C - сообщение или программа СМИ; $\mathrm{P}$ - реципиент

и массовую фрормы связи. В этом состоит ее достоинство. Кроме того, Вестли и Маклин расширили смысл ньюкомбова понятия информационной ориентации. В него включены эмоциональная и рациональная составляющие. сообщение, и поэтому проблема воздействия с точки зрения психологии является частью фактора «реципиент».

К достоинствам модели можно отнести расширенную форму представления процесса передачи со- 
общения через СМИ, а также двустороннее согласование $K$ с $P$.

\section{Математические модели СМИ}

В последнее время в научных журналах появились работы, в которых авторы, используя математический аппарат, а именно дифференциальные уравнения, пытаются описать определенные закономерности развития СМИ как динамической системы. Методы нелинейной динамики дают возможность фрормально и детально изучить процессы, происходящие в этой сложной системе.

При составлении математических моделей принципиальный вопрос заключается в том, какие переменные действительно характеризуют сложную динамическую систему, и как они связаны между собой. Учитывая важность этого вопроса, обратим внимание на некоторые работы.

В статье [19] проведены формализация и анализ информационного обмена в общественных структурах. В рамках данного исследования базовой гипотезой моделирования является предположение: сколь угодно большое, но ограниченное множество $A$ людей (граждан) разделено на группы $X, Y, Z, S$, и существует возможность обмена информацией с помощью СМИ.

В группу Х входят люди, заинтересованные в создании некоторого «сообщества» (например, структуры местного самоуправления, сознательного электората при проведении выборов и т. п.) для достижения поставленной цели. Примерами цели могут служить достижение оптимального функционирования структуры, определенных результатов выборов. При этом участники группы не инфоормированы о способах создания «сообщества», необходимых правовых документах, результатах, экономической и социальной основ и структуры сообщества, полномочиях его исполнительных органов. Группу $Y$ составляют заинтересованные и информированные члены, группу Z - незаинтересованные, но инфрормированные, в группу $S-$ незаинтересованные и неинформированные.

Переменными модели взяты $X(t), Y(t), Z(t), S(t)$ - численность субъектов в соответствующих группах в момент $t$.

Предложив метод информационных балансов при взаимодействии между гражданами различных групп и используя методики постановки нелинейных задач [20; 21], авторами предложена математическая модель изменения численности граждан в группах в процессе информационного обмена:

$$
\begin{aligned}
& \frac{d X}{d t}=-q_{y x} X+Q_{x}+Q_{x c}(t)+ \\
& +b_{y z} Y Z+b_{y s} Y S+b_{z s} Z S
\end{aligned}
$$

$$
\begin{aligned}
& \frac{d Y}{d t}=-q_{y x} Y+q_{y x}\left(Y_{0}+X_{0}\right)- \\
& -\left(Q_{x}+Q_{x c}(t)\right)+q_{y s}(Y-S)+ \\
& +b_{z x} Z X+b_{x S} X S+b_{z S} Z S
\end{aligned}
$$

$$
\begin{aligned}
& \frac{d Z}{d t}=-q_{z s} Z+q_{z s}\left(Z_{0}+S_{0}\right)- \\
& -\left(Q_{z}+Q_{z c}(t)\right)+q_{z x}(Z-X)+ \\
& +b_{y x} Y X+b_{y s} Y S+b_{x S} X S
\end{aligned}
$$

$S=A+A_{c}(t)-X-Y-Z$, 
$X\left(t_{0}\right)=X_{0}, Y\left(t_{0}\right)=Y_{0}, Z\left(t_{0}\right)=$ $=Z_{0}, S\left(t_{0}\right)=S_{0}$.

Здесь $X_{0}, Y_{0}, Z_{0}, S_{0} \geq 0 ; q_{y x}, q_{y s}$, $q_{z s}, q_{z x}, b_{y x}, b_{y s}, b_{x s}, b_{z s}, b_{y s}$ - положительные коэффрициенты, характеризующие интенсивности возрастания, убывания численности в соответствующих группах при поступлении в них информации; $Q_{x}, Q_{z}$ - математическое ожидание скорости изменения числа граждан в группах $X$ и $Y$ и группах $S$ и $Z$ соответственно, благодаря поступлению посторонней информации; $A-$ численность людей в группах в детерминированной постановке или математическое ожидание этой величины, если учитывается случайный характер процессов; $Q_{x c}(t), Q_{z c}(t), A_{c}(t)$ - случайные составляющие соответствующих параметров.

При данных связях между переменными была поставлена задача: путем управляемого оптимизированного информационного обмена между группами максимально увеличить численность заинтересованных и инфрормированных людей $Y \rightarrow Y_{\text {max }}$

Проведенный качественный $и$ количественный анализ задачи (1) (5) позволил сформулировать ряд рекомендаций. К наиболее важным можно отнести следующие:

- необходимо организовать интенсивное информационное взаимодействие группы информированных и незаинтересованных граждан с группой неинформированных и заинтересованных $(Z \leftrightarrow X)$, а также группы информированных и заинтересованных граждан с группой неинформированных и незаинтересованных $(Y \leftrightarrow S)$;
- информационное взаимодействие между группой незаинтересованных и информированных граждан и группой незаинтересованных и неинформированных $(Z \leftrightarrow S)$ не учитывается, так как оно не значимо влияет на достижение желаемого результата $Y \rightarrow Y_{\text {max }}$;

- следует принять возможные меры, направленные на предотвращение больших изменений в общей численности граждан, участвующих в информационном обмене. В противном случае возможно разрушение системы, т.е. переходные процессы теряют устойчивость и поставленная цель $Y \rightarrow Y_{\max }$ не достигается.

В работе [22] процесс информационного обмена в социуме рассмотрен с других позиций и более подробно. На основе базовой модели распространения информации [23] предложены четыре новых механизма, детализирующие протекание процесса:

- контингентное поведение, при котором индивид осознает себя частью группы и его поведение находится в зависимости от величины показателей, характеризующих группу в целом;

- неполный охват социума средствами массовой информации (неоднородность социума);

— забывание информации индивидами;

- двух-шаговое усвоение информации ${ }^{1}$.

В качестве базовой принимается модель, которая описывает процесс распространения нужной информа-

1 Практика показывает, что информационное воздействие результативно, если оно является не одноразовым, а проводится в виде кампании. 
ции в однородном социуме численностью $N$. Предполагается, что неохваченный информацией индивид может получить ее через СМИ либо путем межличностной коммуникации от индивида, владеющего нужной информацией и распространяющего ее (адепта). Модель имеет вид задачи Коши для нелинейного дифференциального уравнения:

$$
\begin{aligned}
& \frac{d X}{d t}=(\alpha+\beta X)(N-X), \\
& X(0)=0
\end{aligned}
$$

где $X(t)$ - численность адептов в момент времени $t$, положительные параметры $\alpha, \beta$ характеризуют интенсивности распространения информации через СМИ и путем межличностной коммуникации соответственно.

Из (6) видно, что скорость распространения информации - число охваченных индивидов в единицу времени - пропорциональна числу еще неохваченных индивидов ( $N-$ $X)$.

Добавление фрактора контингентное поведение предполагает, что индивид является членом некоторой группы. Он может стать адептом, распространять информацию дальше лишь в том случае, если определенное число членов группы, называемое порогом данного индивида, уже являются адептами. Тогда уравнение для численности адептов будет иметь вид:

$$
\frac{d X}{d t}=(\alpha+\beta X)(f(X)-X),
$$

где $f(X)$ характеризуется как функция, описывающая численность людей, которые уже являются адептами либо готовы к ним присоединиться, ориентируясь на текущее значение $X(t)$.

При рассмотрении другой надстройки над базовой моделью предполагается, что рассматриваемая группа состоит из двух однородных подгрупп. Члены первой подгруппы численности $N_{1}$ могут получать информацию как от СМИ, так и через слухи. Вторая подгруппа численности $\mathrm{N}_{2}$ не охвачена СМИ. Она получает информацию только вторым способом.

После обозначения количества адептов первой подгруппы в момент времени $t$ за $X_{1}(t)$, второй - за $X_{2}(t)$, модель (6) усложняется до системы:

$$
\begin{aligned}
& \frac{d X_{1}}{d t}=\left(\alpha+\beta\left(X_{1}+X_{2}\right)\right) \times \\
& \times\left(N_{1}-X_{1}\right) \\
& \frac{d X_{2}}{d t}=\beta\left(X_{1}+X_{2}\right)\left(N_{2}-X_{2}\right) .
\end{aligned}
$$

В базовой модели предполагалось, что адепты не забывают информацию, т.е. не могут вернуться в разряд неохваченных. Отказ от этого условия приводит к системе вида

$$
\begin{aligned}
& \frac{d X_{1}}{d t}=\left(\alpha+\beta\left(X_{1}+X_{2}\right)\right) \times \\
& \times\left(N_{1}-X_{1}\right)-\gamma X_{1},
\end{aligned}
$$

$$
\begin{aligned}
& \frac{d X_{2}}{d t}=\beta\left(X_{1}+X_{2}\right)\left(N_{2}-X_{2}\right)- \\
& -\gamma X_{2},
\end{aligned}
$$

где $y>0$ - интенсивность забывания информации индивидами. 
Далее, в рамках модели (6) предполагается, что индивид присоединяется к числу адептов в два этапа. Будучи охваченным в первый раз, он становится предадептом. Это, в частности, означает, что далее он информацию не распространяет. Обозначив через $x(t)$ численность предадептов, уравнение (6) можно разбить на два:

$$
\begin{aligned}
& \frac{d X}{d t}=(\alpha+\beta X) x, \\
& \frac{d x}{d t}=(\alpha+\beta X)(N-X-x)- \\
& -(\alpha+\beta X) x=(\alpha+\beta X) \times \\
& \times(N-X-2 x) .
\end{aligned}
$$

Тогда с учетом всех четырех факторов базовая модель (6) может быть представлена в виде следующей задачи Коши:

$$
\begin{aligned}
& \frac{d X_{1}}{d t}=\left(\alpha+\beta\left(X_{1}+X_{2}\right)\right) \\
& \left(f_{1}\left(X_{1}+X_{2}\right) \frac{x_{1}+X_{1}}{N_{1}}-X_{1}\right)- \\
& -\gamma X_{1} \\
& \frac{d X_{2}}{d t}=\beta\left(X_{1}+X_{2}\right) \times \\
& \times\left(f_{2}\left(X_{1}+X_{2}\right) \frac{x_{2}+X_{2}}{N_{2}}-X_{2}\right)- \\
& -\gamma X_{2}, \\
& \frac{d x_{1}}{d t}=\left(\alpha+\beta\left(X_{1}+X_{2}\right)\right) \times \\
& \times\left(N_{1}-x_{1}-f_{1}\left(X_{1}+X_{2}\right) \frac{x_{1}+X_{1}}{N_{1}}\right)- \\
& -\delta x_{1}+\gamma X_{1},
\end{aligned}
$$

$$
\begin{aligned}
& \frac{d x_{2}}{d t}=\beta\left(X_{1}+X_{2}\right) \times \\
& \times\left(N_{2}-x_{2}-f_{2}\left(X_{1}+X_{2}\right) \frac{x_{2}+X_{2}}{N_{2}}\right)- \\
& -\delta x_{1}+\gamma X_{2} \\
& X_{1}(0)=X_{2}(0)=x_{1}(0)= \\
& =x_{2}(0)=0
\end{aligned}
$$

Данная модель, в частности, имеет стационарный режим

$$
\begin{aligned}
& X_{1}=X_{2}=0, x_{1}=N_{1} \frac{\alpha}{\alpha+\delta}, \\
& x_{2}=0,
\end{aligned}
$$

который можно интерпретировать как безадептный.

Качественный анализ СМИ показал, что устойчивости этого режима способствуют высокие интенсивности забывания $\delta$ и $ү$ и малая скорость увеличения фрункции $f_{1}(u)$ в начальный момент времени. Асимптотически устойчивое безадептное состояние может быть охарактеризовано следующим образом: ввиду отсутствия адептов в нем не осуществляется передача информации с помощью слухов.

Часть членов первой подгруппы является предадептами, их численность не растет ввиду того, что пополнение класса предадептов компенсируется забыванием. При этом барьер контингентности и «забывчивость» адептов не позволяют сформировать класс адептов.

Во второй подгруппе класс предадептов не фрормируется, поскольку члены этой подгруппы получают инфрормацию только через слухи, для появления которых наличие адептов необходимо. Тот же каче- 
ственный анализ показал, что при фриксированных параметрах забывания и интенсивности СМИ для исхода распространения информации ключевую роль играет скорость увеличения функции $f_{1}(u)$ в начальный момент времени. Это приводит к ненулевой численности адептов, а, следовательно, ведет к возможности доносить нужную информацию в социум.

Рассмотренные работы объединяет общий подход к построению моделей: имитируется процесс распространения информации в обществе через сорормированные СМИ с развитой структурой. Это стало возможным из-за использования в моделях в качестве переменных показатели численности определенных групп реципиентов.

Иной взгляд при моделировании представлен в работе [24], в которой авторы рассматривают СМИ как структуру, развивающуюся эволюционно. СМИ являются особой областью производственной деятельности, которая действует одновременно на рынке товаров и услуг, причем одной из ключевых особенностей является предоставление рекламодателям доступа к массовой или специализированной аудитории. Именно структура рекламного рынка отражает изменения, которые происходят в медиаиндустрии, поскольку зависимость стоимости рекламного времени обусловлена привлекательностью для аудитории того или иного медиаканала (печатная продукция, радио, телевидение, интернет). Данный фракт привел к возможности связать изменения в структуре СМИ с изменениями на рынке рекламы. В данной работе представлена попытка фрор- мально описать динамику развития медиаканалов с помощью системы обыкновенных дифференциальных уравнений, фазовыми переменными которой являются объемы вложений в рекламу.

Пусть на временном промежутке $\left[T_{0}, T_{N-1}\right]$ в моменты $T_{0}, T_{1}, \ldots, T_{\mathrm{N}-1}$ последовательно возникают новые каналы СМИ с номерами 1, 2,.., $N$, а $x_{k}(t), k=1,2, \ldots, N$ - рекламный бюджет $k$-го медиаканала. Каждый новый канал СМИ (кроме самого первого) изымает в свою пользу у всех остальных с меньшими номерами некоторую долю рекламных бюджетов, равную

$$
\beta_{k}^{*} \sum_{i=0}^{k-1} x_{i}, 0<\beta_{k}^{*}<1
$$

и использует эту долю в качестве своего ресурса. Тогда на интервале $\left[T_{k-1}, T_{k}\right]$ уравнение, описывающее поведение определенного канала СМИ в фразе его развития, т. е. до появления нового медиаканала, начинающего изымать у него часть рекламного бюджета, будет иметь вид:

$$
\dot{x}_{k}=a_{k} x_{k}\left(1-\frac{x_{k}}{h_{k}}\right)+\beta_{k}^{*} \sum_{i=0}^{k-1} x_{i}
$$

Здесь $\alpha_{k}$ - интенсивность, а $h_{k}$ - потенциал данного канала СМИ. В этих условиях $\beta_{k}$ - доля совокупного продукта первых $(k-1)$ каналов, изымаемая в пользу $k$-ого канала СМИ. Естественно положить

$$
\beta_{1}^{*}=0, \quad x_{0}(t) \equiv 0
$$

После возникновения нового медиаканала, согласно предположению, канал $k$ уже сам отдает ему 
часть продукта $\beta_{k+1}^{*}$. Поэтому уравнение приобретает вид:

$$
\begin{aligned}
& \dot{x}_{k}=a_{k}\left(1-\beta_{k+1}^{*}\right) x_{k} \\
& 1-\frac{x_{k}}{h_{k}}-\frac{1}{a_{k}} \frac{\beta_{k+1}^{*}-\beta_{k+1}^{*}}{k-1}+ \\
& +\beta_{k}^{*}\left(1-\beta_{k+1}^{*}\right)_{i=0}^{k} x_{i}, \\
& T_{k} \leq t \leq T_{k+1}
\end{aligned}
$$

Обобщая рассуждения, получен общий вид системы обыкновенных диффреренциальных уравнений, описывающих поведение совокупности $N$ медиаканалов на промежутке $\left[T_{0}, T_{N}\right]:$

$$
\begin{aligned}
& \dot{x}_{k}=a_{k} R_{k}{ }^{x} k \\
& \left(1-\frac{x_{k}}{h_{k}}-\frac{S_{k}}{a_{k}}\right)+ \\
& +\beta_{k}^{*} R_{k} \sum_{i=0}^{k-1} x_{i}, \quad k=1, \ldots, N
\end{aligned}
$$

при начальных условиях

$x_{1}\left(T_{0}\right)=C_{0}>0, \quad x_{k}\left(T_{0}\right)=0, \quad k \neq 1$.

$$
\begin{aligned}
& \text { Здесь } \beta_{k}= \begin{cases}0, & t \leq T_{k-1}, \\
\beta_{k}^{*}, & t>T_{k-1} ;\end{cases} \\
& R_{k}=\stackrel{M}{I=k+1}_{i=k+2}^{N}\left(1-2 \beta_{i}\right) ;
\end{aligned}
$$

$$
S_{k}=\sum_{i=k+1}^{N} \frac{2 \beta_{i}}{\prod_{j=k+1}^{i}\left(1-2 \beta_{j}\right)}
$$

Таким образом, динамика каналов СМИ описана следующими наборами параметров:

- общим числом медиаканалов на заданном временном интервале и моментами их возникновения;

- интенсивностями и потенциалами медиаканалов;

- коэффицциентами изъятия фринансирования старых медиаканалов более молодыми;

- полным объемом рекламного рынка в момент отсчета $T_{0}$.

Признаком возникновения нового медиаканала считается ситуация, в которой медиаканал с номером фрактически прекращает свой рост, как бы исчерпывая свой «потенциал эффективности». Формально это означает, что

$\frac{d x_{k}\left(T_{k}\right)}{d t}=\varepsilon$,

где $\varepsilon$ - достаточно малая положительная величина. В это время и возникает новый, $(k+1)$-ый канал СМИ.

Условием, позволяющим связывать медиаканалы с расходами на рекламу, является предположение, что в момент возникновения нового канала СМИ сумма рекламных расходов всех медиаканалов совпадает с известной величиной расходов на рекламу.

$$
\sum_{i=1}^{N} x_{i}\left(T_{k}\right)=C_{k}>0, \quad k=1, \ldots, N .
$$


Среди задач, которые можно решить с помощью модели (7)-(10), наибольший интерес, по мнению авторов, представляет следующая: подобрать параметры развития медиаканалов так, чтобы получаемая при этом расчетная траектория темпов роста суммарных рекламных расходов была достаточно близка к фактической. В качестве критериев близости предлагаются два:

- максимум коэффициента корреляции между фрактической и расчетной траекториями темпов роста расходов на рекламу;

- минимум суммы квадратов погодовых отклонений расчетной траектории темпов расходов от фактической.

Заключение. Приведенные концептуальные формы организации СМИ выявили широкий спектр подходов в описании процессов коммуникации. Диапазон решений простирается от упрощенной статической конструкции связи между источником и потребителем информации до описаний процессов формирования структуры как самих СМИ, так и сообщества потребителей продукта СМИ (активных и пассивных) на языке диффреренциальных уравнений (в динамическом аспекте).
Различие позиций моделирования раскрывает предмет исследования с противоположных сторон, обе полезны для представления реальной медиасистемы. С одной стороны, механистический способ моделирования по сути является гомоморфным описанием, с другой, математическое аналоговое представление фрормирования структуры реципиентов усложнен и тяготеет к изоморфному отражению.

Прогресс современного научного познания требует органичного сочетания подходов для взаимодействия и методологического синтезирования в целях решения насущных задач управления социальными системами. Как известно, степень сложности описания предмета исследования обратна степени понимания результата моделирования. В целях раскрытия принципов управления информационными процессами следует, на наш взгляд, использовать когнитивный подход. Для адекватного восприятия и практического применения математических моделей необходимо, чтобы современный математический аппарат не затруднял, а наоборот, помогал яснее отображать сущность моделируемого процесса, постановки задач и методы их решения.

\section{СПИСОК ИСПОЛЬЗОВАННОЙ ЛИТЕРАТУРЫ}

1. Богданов А. А. Тектология (Всеобщая организационная наука) : в 2 кн. / А. А. Богданов. - М. : Экономика, 1989. - Кн. 1. - 304 с.

2. Берталанфи Л. фон. Общая теория систем: критический обзор / Л. фон Берталанфи // Исследования по общей теории систем : сб. переводов / общ. ред. В. Н. Садовского, Э. Г. Юдина. - М. : Прогресс, 1969. - С. 23-82.

3. Нейлор Т. Машинные имитационные эксперименты с моделями экономических систем : пер. с англ. / Т. Нейлор. - М. : Мир, 1975. - 499 с.

4. Шеннон Р. Имитационное моделирование систем: искусство и наука : пер. с англ. / Р. Шеннон. - М. : Мир, 1978. - 418 с.

5. Шеннон К. Работы по теории информации и кибернетике / К. Шеннон. - М. : Иностр. лит., 1963. - 830 с. 
6. Кузнецова И. А. Гомеостатическая модель конкурентных отношений в социальноэкономической системе : дис. ... канд. техн. наук : 05.13.01 / И. А. Кузнецова. — Иркутск, 2006. $-188 \mathrm{c}$.

7. Shannon C. E. A mathematical theory of communication / C. E. Shannon // Bell System Technical Journal. - 1948. - Vol. 27. - P. 379-423.

8. Lasswell H. D. Propaganda Technique in the World War / H. D. Lasswell. - London ; New York : Peter Smith Publ., 1927. - 233 p.

9. Propaganda and Communication in World History: in 3 vols / eds.: H. D. Lasswell, D. Lerner, H. Speier. - Hawaii : University of Hawaii Press, 1980.

10. Эшби У. Р. Введение в кибернетику : пер с англ. / У. Р. Эшби. - М. : Иностр. лит., 1959. -432 c.

11. Горский Ю. М. Системно-информационный анализ процессов управления / Ю. М. Горский. - Новосибирск : Наука, 1988. - 327 с.

12. Прангишвили И. В. Системный подход и общесистемные закономерности / И. В. Прангишвили. - М. : Синтег, 2000. - 528 с.

13. Гомеостатика живых, технических, социальных и экологических систем / Ю. М. Горский, В. И. Астафьев, В. П. Казначеев [и др.]. - Новосибирск : Наука, 1990. - 350 с.

14. Горский Ю. М. Гомеостатика: гармония в игре противоречий / Ю. М. Горский, А. М. Степанов, А. Г. Теслинов. - Иркутск : Репроцентр А1, 2008. - 634 с.

15. Назаров М. М. Массовая коммуникация в современном мире: методология анализа и практика исследований / М. М. Назаров. - М. : Эдиториал УРСС, 2003. 239 c.

16. McQuail D. Mass Communication Theory: An Introduction / D. McQuail D. - 2nd ed. - Beverly Hills, CA : Sage Publ., 1987. - 448 p.

17. Гавра Д. Основы теории коммуникации : учеб. пособие / Д. Гавра. - СПб. : Питер, 2011. - 288 с.

18. Maletzke G. Psychologie der Massen-Kommunikation. Theorie und Systematik / G. Maletzke. - Hamburg : Verlag Hans Bredow-Institut, 1963. - $311 \mathrm{~s}$.

19. Математическое моделирование и управление в системах информирования и информационного обмена в обществе / В. Э. Джашитов, В. М. Панкратов, А. Ф. Резчиков, А. Э. Джашитов // Проблемы управления. - 2009. - № 6. - С. 2-8.

20. Леонов Г. А. Динамические принципы прогнозирования и управления / Г. А. Леонов // Проблемы управления. - 2008. — № 5. - С. 31-35.

21. Вышкинд С. А. О динамике некоторых математических моделей в социологии / С. А. Вышкинд, А. А. Деветьярова // Известия вузов. Сер.: Прикладная нелинейная динамика. - 1994. - № 2. - С. 17-26.

22. Развитие модели распространения информации в социуме / А. П. Михайлов, А. П. Петров, Н. А. Маревцева, И. В. Третьякова // Математическое моделирование. 2014. - Т. 26, № 3. - С. 65-74.

23. Самарский А. А. Математическое моделирование / А. А. Самарский, А. П. Михайлов. - М. : Физматлит, 2006. - 320 с.

24. Баенхаева А. В. Эволюционный подход к развитию средств массовой информации: построение математической модели / А. В. Баенхаева, С. В. Тимофеев // Известия Байкальского государственного университета. — 2016. - Т. 26, №5. - С. 825-833.

\section{REFERENCES}

1. Bogdanov A. A. Tektologiya (Vseobshchaya organizatsionnaya nauka) [Textology (Universal Organizing Science)]. Moscow, Ekonomika Publ., 1989. Vol. 1. 304 p.

2. Bertalanffy L. von. General System Theory - A Critical Review. General Systems. 1962, vol. 7, pp. 1-20. (Russ. ed.: Bertalanffy L. von. Obshchaya teoriya sistem: kriticheskii obzor. In Sadovskii V. N., Yudina E. G. (eds). Issledovaniya po obshchei teorii sistem. Moscow, Progress Publ., 1969, pp. 23-82.).

3. Neilor T. Mashinnye imitatsionnye eksperimenty s modelyami ekonomicheskikh sistem [Machine Imitation Experiments with Economic Systems' Models]. Moscow, Mir Publ., 1975. 499 p. 
4. Shannon R. Systems Simulation. The Art and Science. Prentice-Hall, 1975. (Russ. ed.: Shannon R. Imitatsionnoe modelirovanie sistem: iskusstvo i nauka. Moscow, Mir Publ., 1978. 418 p.).

5. Shannon K. Raboty po teorii informatsii i kibernetike [Works on Theory of Information and Cybernetics]. Moscow, Inostrannaya literatura Publ., 1963. 830 p.

6. Kuznetsova I. A. Gomeostaticheskaya model' konkurentnykh otnoshenii v sotsial'noekonomicheskoi sisteme. Kand. Diss. [A Homeostasis Model of Competitive Relations in a Socio-Economic System. Cand. Diss.]. Irkutsk, 2006. 188 p.

7. Shannon C. E. A mathematical theory of communication. Bell System Technical Journal, 1948, vol. 27, pp. 379-423.

8. Lasswell H. D. Propaganda Technique in the World War. London, New York, Peter Smith, 1927. 233 p.

9. Lasswell H.D., Lerner D., Speier H. (eds). Speier Propaganda and Communication in World History: in 3 vols. University of Hawaii Press, 1980.

10. Ashby W. R. An Introduction to Cybernetics. Chapman and Hall, 1957. (Russ. ed.: Ashby W. R. Vvedenie v kibernetiku. Moscow, Inostrannaya literatura Publ., 1959. 432 p.).

11. Gorskii Yu. M. Sistemno-informatsionnyi analiz protsessov upravleniya [The Systemic and Information Analysis of Management Processes]. Novosibirsk, Nauka Publ., 1988. 327 p.

12. Prangishvili I. V. Sistemnyi podkhod i obshchesistemnye zakonomernosti [The Systemic Approach and Systemic Laws]. Moscow, Sinteg Publ., 2000. 528 p.

13. Gorskii Yu. M., Astaf'ev V. I., Kaznacheev V. P. et al. Gomeostatika zhivykh, tekhnicheskikh, sotsial'nykh i ekologicheskikh sistem [Homeostatics of Living, Technical, Social and Economic Systems]. Novosibirsk, Nauka Publ., 1990. 350 p.

14. Gorskii Yu. M., Stepanov A. M., Teslinov A. G. Gomeostatika: garmoniya v igre protivorechii [Homeostatics: Harmony in a Play of Contradictions]. Irkutsk, Reprotsentr A1, 2008. $634 \mathrm{p}$.

15. Nazarov M. M. Massovaya kommunikatsiya $v$ sovremennom mire: metodologiya analiza i praktika issledovanii [Mass Communication in Modern Society: Methodology of Analysis and Research Experience]. Moscow, Editorial URSS Publ., 2003. 239 p.

16. McQuail D. Mass Communication Theory: An Introduction. 2nd ed. Beverly Hills, CA, Sage Publ., 1987. 448 p.

17. Gavra D. Osnovy teorii kommunikatsii [Basics of the Theory of Communication]. Saint Petersburg, Piter Publ., 2011. 288 p.

18. Maletzke G. Psychologie der Massen-Kommunikation. Theorie und Systematik. Hamburg, Verlag Hans Bredow-Institut, 1963. 311 s.

19. Dzhashitov V. E., Pankratov V. M., Rezchikov A. F., Dzhashitov A. E. Mathematical modeling and control in social informing and information exchange systems. Problemy upravleniya $=$ Problems of Management, 2009, no. 6, pp. 2-8. (In Russian).

20. Leonov G. A. Dynamic principles of prediction and control. Problemy upravleniya = Problems of Management, 2008, no. 5, pp. 31-35. (In Russian).

21. Vyshkind S. A., Devet'yarova A. A. On Dynamics of Some Mathematical Models in Socilology. Izvestiya Vuzov. Seriya: Prikladnaya nelineinaya dinamika = Applied Nonlinear Dynamics. Izvestiya Vuz, 1994, no. 2, pp. 17-26. (In Russian).

22. Mikhailov A. P., Petrov A. P., Marevtseva N. A., Tret'yakova I. V. Development of a model of information dissemination in society. Matematicheskoe modelirovanie = Mathematical Models and Computer Simulations, 2014, vol. 26, no. 3, pp. 65-74. (In Russian).

23. Samarskii A. A., Mikhailov A. P. Matematicheskoe modelirovanie [Mathematical Models]. Moscow, Fizmatlit Publ., 2006. 320 p.

24. Baenkhaeva A. V., Timofeev S. V. The evolutionary approach to development of mass media: construction of a mathematical model. Izvestiya Baykal'skogo gosudarstvennogo universiteta = Bulletin of Baikal State University, 2016, vol. 26, no. 5, pp. 825-833. DOI: 10.17150/2500-2759.2016.26(5).825-833 (In Russian). 
Вопросы теории и практики журналистики. 2017. Т. 6. № 3. С. 287-305

\section{БИБЛИОГРАФИЧЕСКОЕ ОПИСАНИЕ СТАТЬИ}

Суходолов А. П. Анализ подходов в моделировании средств массовой информации / А. П. Суходолов, И. А. Кузнецова, С. В. Тимофеев // Вопросы теории и практики журналистики. — 2017. — T. 6, № 3. — C. 287-305. DOI: 10.17150/2308-6203.2017.6(3).287-305.

\section{REFERENCE TO ARTICLE}

Sukhodolov A. P., Kuznetsova I. A., Timofeev S. V. The Analysis of Approaches in Modelling of Mass Media. Voprosy teorii $i$ praktiki zhurnalistiki $=$ Theoretical and Practical Issues of Journalism, 2017, vol. 6, no. 3, pp. 287-305. DOI: 10.17150/2308-6203.2017.6(3).287-305. (In Russian). 